Ergod. Th. \& Dynam. Sys. (1988), 8*, 139-152

Printed in Great Britain

\title{
Four applications of conformal equivalence to geometry and dynamics
}

\author{
ANATOLE KATOK \\ Mathematics 253-37, California Institute of Technology, Pasadena, CA 91125, USA
}

Abstract. Conformal equivalence theorem from complex analysis says that every Riemannian metric on a compact surface with negative Euler characteristics can be obtained by multiplying a metric of constant negative curvature by a scalar function. This fact is used to produce information about the topological and metric entropies of the geodesic flow associated with a Riemannian metric, geodesic length spectrum, geodesic and harmonic measures of infinity and Cheeger asymptotic isoperimetric constant. The method is rather uniform and is based on a comparison of extremals for variational problems for conformally equivalent metrics.

\section{0 . Introduction}

Let $M$ be a compact surface with negative Euler characteristic $E$ and let $\sigma$ be a smooth, say $C^{\infty}$, Riemannian metric on $M$.

Classical regularization theorems from complex analysis (see e.g. [15]) imply the following fact.

CONFORMAL EQUIVALENCE THEOREM. There exists a scalar positive $C^{\infty}$ function $\rho$ on $M$, uniquely defined up to a positive constant, such that the Riemannian metric po has constant negative curvature.

Two metrics $\sigma, \sigma^{\prime}$ which differ by a scalar function (i.e. $\sigma^{\prime}=\rho \sigma$ ) determine the same complex structure on $\boldsymbol{M}$. We will call such metrics conformally equivalent. Sometimes they are also called conformal.

The conformal equivalence theorem was used in [8] to obtain estimates for the entropies (topological and metric with respect to Liouville measure) of the geodesic flow associated with a Riemannian metric and for the growth rate of the number of closed geodesics. In this note we show that similar arguments produce some information about other important characteristics of a Riemannian metric on a surface, namely the geodesic length spectrum, geodesic and harmonic measures at infinity, and the Cheeger asymptotic isoperimetric constant [3]. To emphasize the similarity of the arguments in all cases, we begin with a slightly different and more easily accessible presentation of one of the main results from [8] and then proceed to new applications of the conformal equivalence theorem.

I would like to point out that I learnt about the Cheeger constant from my Caltech colleague Michael Anderson. He proved that for an arbitrary metric the Cheeger constant does not exceed the topological entropy and conjectured that for a metric of negative curvature it is greater than or equal to the Liouville entropy (see $\$ 5$ below). For the surface case theorem 5 gives a much better upper estimate for the 
Cheeger constant than topological entropy. This theorem came as an aftermath of several discussions with Anderson and should be considered as a joint result.

\section{Entropy estimates}

Throughout this paper we will use the same notations as in [8]. In particular, let $h_{\sigma}$ be the topological entropy of the geodesic flow $\phi^{\sigma}=\left\{\phi_{t}^{\sigma}\right\}_{t \in \mathbb{R}}$ associated with the metric $\sigma$ and let $h_{\sigma}^{\lambda}$ be the metric entropy with respect to the smooth invariant measure $\lambda_{\sigma}$ (Liouville measure) on the unit tangent bundle $S^{\sigma} M$. The total area of the surface $M$ will be denoted by $V_{\sigma}$ and the normalized Riemannian measure on $M$, which coincides with the projection of $\lambda_{\sigma}$ to $M$, by $\mu_{\sigma}$. Furthermore, let us choose the function $\rho$ which comes from the conformal equivalence theorem in such a way that the total areas of $M$ with respect to $\sigma$ and $\rho \sigma$ are the same. Equivalently, this means that $\int \rho d \mu_{\sigma}=1$. We denote the number $\int \rho^{1 / 2} d \mu_{\sigma}$ by $\rho_{\sigma}$ and call it the conformal coefficient of the metric $\sigma$. By the Schwartz inequality, $\rho_{\sigma} \leq 1$ and $\rho_{\sigma}=1$ if and only if $\sigma$ itself is a metric of constant negative curvature.

For a metric $\sigma_{0}$ of constant negative curvature $-K^{2}$ one has (see [8], (3.21))

$$
h_{\sigma_{0}}^{\lambda}=h_{\sigma_{0}}=K=\left(-2 \pi E / V_{\sigma_{0}}\right)^{1 / 2} \text {. }
$$

The following theorem summarizes those of the results of [8] for surfaces, which are based on the conformal equivalence theorem. We reproduce the complete formulation, although we will prove here only a part of the result.

THEOREM 1 (see [8], theorems 3.1 and 3.2). For any metric $\sigma$ on $M$

$$
h_{\sigma} \geq \rho_{\sigma}^{-1}\left(-2 \pi E / V_{\sigma}\right)^{1 / 2}
$$

and this inequality is strict unless $\sigma$ is a metric of constant negative curvature.

If, moreover, $\sigma$ is a metric without focal points (see [10]; e.g. if it has non-positive curvature), then

$$
h_{\sigma}^{\lambda} \leq \rho_{\sigma}\left(-2 \pi E / V_{\sigma}\right)^{1 / 2}
$$

and this inequality is also strict for any metric of non-constant curvature.

We will give here a proof only for the non-strict version of (2). The argument presented below preceded the one given in [8] and is very suggestive in view of other applications of the conformal equivalence theorem discussed in $\$ \S 2,3$ and 4. The non-strict version of (3) for a metric of negative curvature can be obtained in a very similar way. Making the last inequality work for metrics without focal points, as well as showing that the inequalities are strict, requires slightly more subtle considerations which actually somewhat obscure the main idea, namely comparing the extremals of a variational problem (in this case, closed geodesics) for conformally equivalent metrics.

Proof. Take the metric of constant negative curvature $\sigma_{0}=\rho \sigma$, where as before $V_{\sigma_{0}}=V_{\sigma}$. Fix a large number $T$ and consider all closed geodesics for $\sigma_{0}$ of length $\leq T$. The total number $P_{\sigma_{0}}(t)$ of such geodesics satisfies the asymptotic formula

$$
\lim _{T \rightarrow \infty} \frac{P_{\sigma_{0}}(T)}{h_{\sigma_{0}}^{-1} T^{-1} \exp \left(T h_{\sigma_{0}}\right)}=1 \text {. }
$$


For metrics of constant negative curvature this asymptotic follows from the Selberg trace formula for variable negative curvature from the work of Margulis [13].

Actually, we do not even need this precise multiplicative asymptotic for $P_{\sigma_{0}}(T)$, but only a weaker exponential asymptotic (see e.g. [1])

$$
\lim _{T \rightarrow \infty} \frac{\log P_{\sigma_{0}}(T)}{T}=h_{\sigma_{0}} \text {. }
$$

In addition to that, we use the fact that Liouville measure $\lambda_{\sigma_{0}}$ is the unique measure with maximal entropy for the geodesic flow $\phi^{\sigma_{0}}$. This implies that for a large $T$ the majority of closed geodesics of length $\leq T$ are almost uniformly distributed with respect to $\lambda_{\sigma_{0}}$.

More precisely, we claim that for any continuous test function $f$ on $S^{\sigma_{0}} M$ and for any $\varepsilon>0$, among all closed geodesics of length $\leq T$, the proportion of those for which the average of $f$ along the geodesic is within $\varepsilon$ of the space average $\int_{S^{\sigma_{0}}} f d \lambda_{\sigma_{0}}$ goes to one. For, if we assume the contrary, then there exist a sequence $T_{n} \rightarrow \infty$, a continuous function $f$ and a number $\varepsilon_{0}>0$ such that there are more than $\varepsilon_{0} P_{\sigma_{0}}\left(T_{n}\right)$ geodesics $\gamma$ of length $\leq T_{n}$ for which $f_{\gamma}>\int_{S^{\sigma_{0}}{ }_{M}} f d \lambda_{\sigma_{0}}+\varepsilon_{0}$ (or $f_{\gamma}<\int_{S^{\sigma_{0}}{ }_{M}} f d \lambda_{\sigma_{0}}-\varepsilon_{0}$ ). Here $f_{\gamma}$ denotes the average of $f$ along $\gamma$.

Consider the first case. Take the average $\mu_{n}$ of uniform $\delta$-measures on all our geodesics. Then

$$
\int_{S^{\sigma_{0} M}} f d \mu_{n}>\int_{S^{\sigma_{0}}} f d \lambda_{\sigma_{0}}+\varepsilon_{0} .
$$

But the argument from $[6, \S 4]$ shows that for any weak limit point $\mu$ of the sequence $\mu_{n}$

$$
h_{\mu}\left(\phi^{\sigma_{0}}\right) \geq \lim _{n \rightarrow \infty} \frac{\log \varepsilon_{0} P_{\sigma_{0}}\left(T_{n}\right)}{T_{n}}=h_{\sigma_{0}} .
$$

Since by (4) $\mu \neq \lambda_{\sigma_{0}}$, we obtain a contradiction.

Thus let us fix $\varepsilon>0$ and assume that $T$ is large enough, so that for at least $(1-\varepsilon) P_{\sigma_{0}}(T)$ of all closed geodesics of length $\leq T$ the averages of the function $\rho^{-1 / 2}$ are within $\varepsilon$ of the average of that function with respect to $\lambda_{\sigma_{0}}$. The last average is equal to $\rho_{\sigma}$ because

$$
\int_{S^{\sigma_{0} M}} \rho^{-1 / 2} d \lambda_{\sigma_{0}}=\int_{M} \rho^{-1 / 2} d \mu_{\sigma_{0}}=\int_{M} \rho^{1 / 2} \rho^{-1} d \mu_{\sigma_{0}}=\int_{M} \rho^{1 / 2} d \mu_{\sigma}=\rho_{\sigma} .
$$

The $\sigma$-length of any $\sigma_{0}$-closed geodesic $\gamma$ is equal to its $\sigma_{0}$-length multiplied by the average of the function $\rho^{-1 / 2}$ along $\gamma$. Thus for any of our $\sigma_{0}$-geodesics the $\sigma$-length is going to be less than $T\left(\rho_{\sigma}+\varepsilon\right)$, while their total number is at least $(1-\varepsilon) P_{\sigma_{0}}(T)$.

Each of these geodesics represents a different free homotopy class and each of those classes contains a closed geodesic with respect to $\sigma$. This geodesic is the shortest $\sigma$-curve in its free homotopy class, so that it is not longer than the $\sigma_{0}$-closed geodesic and consequently is shorter than $T\left(\rho_{\sigma}+\varepsilon\right)$.

For an arbitrary metric $\sigma$ let us denote by $P_{\sigma}(T)$ the number of free homotopy classes whose shortest representatives are no longer than $T$. Thus we have shown that

$$
P_{\sigma}\left(T\left(\rho_{\sigma}+\varepsilon\right)\right) \geq(1-\varepsilon) P_{\sigma_{0}}(T) .
$$


Since closed geodesics from different homotopy classes are $\delta_{0}$-separated, where $\delta_{0}$ depends only on the metric, we have from (6)

$$
h_{\sigma} \geq \varlimsup_{T \rightarrow \infty} \frac{\log P_{\sigma}\left(T\left(\rho_{\sigma}+\varepsilon\right)\right)}{T\left(\rho_{\sigma}+\varepsilon\right)} \geq \varlimsup_{T \rightarrow \infty} \frac{\log (1-\varepsilon) P_{\sigma_{0}}(T)}{T\left(\rho_{\sigma}+\varepsilon\right)}=\left(\rho_{\sigma}+\varepsilon\right)^{-1} h_{\sigma_{0}} .
$$

Since $\varepsilon$ can be chosen arbitrarily small,

$$
h_{\sigma} \geq \rho_{\sigma}^{-1} h_{\sigma_{0}} \text {. }
$$

An alternative way to find enough closed geodesics, which are almost uniformly distributed with respect to $\lambda_{\sigma_{0}}$ (or to any given invariant ergodic measure $\mu$ with positive entropy), is to use the definition of metric entropy through separated sets and a closing lemma (see [8], 1.6 and 1.3). This approach yields at least $\exp T\left(h_{\mu}\left(\phi^{\sigma}\right)-\varepsilon\right)$ almost $\mu$-uniformly distributed closed geodesics for any metric $\sigma$ of negative curvature. Then the same variational argument as before allows us to obtain (3) by switching the roles of $\sigma$ and $\sigma_{0}$ and using (1).

Next we will show that even a simpler version of the argument indicated above produces another useful result.

\section{Geodesic length spectrum and classification of metrics of negative curvature}

Let $\Gamma$ be the set of all non-zero free homotopy classes of closed curves on $M$. For each $\gamma \in \Gamma$ let $L_{\sigma}(\gamma)$ be the infimum of lengths of curves representing $\gamma$ or, equivalently, the length of the shortest closed geodesics representing $\gamma$. In particular, if $\sigma$ is a metric of negative curvature, then there is only one closed geodesic representing $\gamma$ and, naturally, $L_{\sigma}(\gamma)$ is equal to the length of that closed geodesic.

We will call the function $L_{\sigma}: \Gamma \rightarrow \mathbb{R}_{+}$the geodesic length spectrum of $\sigma$.

Two metrics $\sigma$ and $\sigma^{\prime}$ on $M$ are called strongly isometric if there exists a diffeomorphism $g$ of $M$ onto itself isotopic to identify and such that $g_{*} \sigma=\sigma^{\prime}$.

The conformal equivalence theorem allows one to reduce the classification of metrics up to a strong isometry to two easier problems: one dealing with conformally equivalent metrics and the other with different metrics of curvature -1 . The space of such metrics (Teichmüller space) is finite-dimensional and its structure is well understood.

Conjecture (see [2], conjecture 3.1). If two metrics of negative curvature on a compact surface have the same geodesic length spectrum, then the metrics are strongly isometric.

There is strong evidence in favour of this conjecture which is discussed in [2, § 3]. In particular the following result is true [9].

THEOREM A. If metrics $\sigma, \sigma^{\prime}$ of negative curvature on a compact surface $M$ have the same length spectrum, then there exists a $C^{\infty}$ diffeomorphism isotopic to identity $g: S^{\sigma} M \rightarrow S^{\sigma^{\prime}} M$ such that $\phi_{t}^{\sigma^{\prime}} g=g \phi_{t}^{\sigma}$.

We will use the reduction mentioned above to show that the conjecture is true modulo finitely many parameters.

THEOREM 2. Different conformally equivalent metrics of negative curvature on a compact surface have different geodesic length spectra. 
Proof. Let $\sigma, \sigma^{\prime}$ be two conformally equivalent metrics of negative curvature so that $\sigma^{\prime}=\rho \sigma$. We can always assume that

$$
V_{\sigma^{\prime}} \leq V_{\sigma}, \quad \text { i.e. } \int_{M} \rho d \mu_{\sigma}=\frac{V_{\sigma^{\prime}}}{V_{\sigma}} \leq 1,
$$

and consequently

$$
\int_{M} \rho^{1 / 2} d \mu_{\sigma}<1 \quad \text { unless } \rho \equiv 1 .
$$

Geodesic flow $\phi^{\sigma}$ is ergodic with respect to Liouville measure $\lambda_{\sigma}$. Fix an orbit of that flow for which the Birkhoff ergodic theorem applies for all continuous functions (such an orbit is necessarily dense in $S^{\sigma} M$ ) and take a sufficiently long piece of that orbit which almost comes back. Then, applying the Anosov closing lemma (see e.g. [8], 1.3), one obtains a closed geodesic which is almost uniformly distributed with respect to $\lambda_{\sigma}$. In particular, the average of the function $\rho^{1 / 2}$ over this geodesic is within $\varepsilon$ of the number $\int_{M} \rho^{1 / 2} d \mu_{\sigma}$ and, if $\varepsilon$ is small enough, that average is less than one (cf. (7)). Thus the $\sigma^{\prime}$-length of that geodesic is less than its $\sigma$-length and the $\sigma^{\prime}$-closed geodesic in the same free homotopy class $\gamma$ is even shorter, so that

$$
L_{\sigma} \cdot(\gamma)<L_{\sigma}(\gamma) \text {. }
$$

Remarks. (1) In the proof of theorem 2 we did not use the fact that $\sigma^{\prime}$ is also a metric of negative curvature. However, the choice of $\sigma$ was determined by the inequality $V_{\sigma} \geq V_{\sigma^{\prime}}$. Thus, if we assume from the beginning that two metrics have the same area and that only one of them has negative curvature, they will still have different length spectra.

(2) For metrics of negative curvature the geodesic length spectrum actually determines the area, but this fact is not needed for our proof.

Corollary. A metric of negative curvature on a compact surface is determined up to a strong isometry by the geodesic length spectrum and by finitely many extra parameters, namely Teichmüller coordinates of its conformal type.

Theorem 2 can be strengthened by replacing in its assumption the geodesic length spectrum by a certain part of it. Let $\beta \in H_{1}(M, Z)$ and let $\Gamma_{\beta}$ be the part of $\Gamma$ which consists of free homotopy classes of closed curves homologous to $\beta$. Let us call the restriction of the geodesic length spectrum to $\Gamma_{\beta}$ the $\beta$-length spectrum for $\sigma$.

THEOREM 3. For each $\beta \in H_{1}(M, Z)$ different conformally equivalent metrics of negative curvature on a compact surface have different $\beta$-length spectra.

Proof. If one can find an almost uniformly distributed closed geodesic whose homology class is equal to $\beta$, the rest of the proof of theorem 2 works without any changes. Since the possibility of such a choice is an interesting statement in its own right, we formulate and prove it separately.

LEMMA. Let $\sigma$ be a metric of negative curvature on a compact surface $M, \beta \in H_{1}(M, Z)$, $f_{1}, \ldots, f_{m}$ be continuous functions on $S^{\sigma} M$ and $\varepsilon>0$. There exists a closed geodesic 
for $\sigma$ whose homology class is equal to $\beta$ and such that the average of each function $f_{i}$ over the geodesic is within $\varepsilon$ of the average $\int_{S_{\sigma} M} f_{i} d \lambda_{\sigma}, i=1, \ldots, m$.

Proof. Let us choose a collection of differential 1-forms $\omega_{1}, \ldots, \omega_{p}$ on $M$ whose cohomology classes form a basis in the first cohomology group $H^{1}(M, \mathbb{R})$. Each form $\omega_{i}$ can be considered as a function on the tangent bundle $T M$. Let us denote the restriction of that function to the unit tangent bundle $S^{\sigma} M$ by $\Omega_{i}$. Since for every $v \in S^{\sigma} M, \Omega_{i}(-v)=-\Omega_{i}(v)$ and the 'flip' operation which sends $v$ to $-v$ preserves Liouville measure, we have

$$
\int_{S^{\sigma} M} \Omega_{i} d \lambda_{\sigma}=0 .
$$

Thus, by choosing a sufficiently long, almost uniformly distributed closed geodesic $g$ as in the proof of theorem 2 , one can assume that the averages of the functions $\Omega_{1}, \ldots, \Omega_{p}$ over $g$ are less than $\varepsilon_{1}$ in absolute value. The constant $\varepsilon_{1}$ will be chosen later and will depend only on $\sigma, f_{1}, \ldots, f_{m}$ and $\varepsilon$.

In addition we can assume that the geodesic $g$ is $\delta$-dense, i.e. that it intersects every $\delta$-ball in $S^{\sigma} M$. Here $\delta$ will also be specified later and will depend only on the given data.

The homology group $H_{1}(M, Z)$ is naturally represented as the integer lattice in $H_{1}(M, \mathbb{R})$ if $M$ is orientable, and as the direct product of that lattice with a cyclic group of order two if $M$ is non-orientable. Let us define a 'norm' for $\alpha \in H_{1}(M, Z)$ as follows:

$$
\|\alpha\|=\sum_{i=1}^{m}\left|\int_{\alpha} \omega_{i}\right|+\Delta,
$$

where $\Delta=0$ if $M$ is orientable or if $\alpha$ belongs to the lattice part of $H_{1}(M, Z)$, and $\Delta=1$ otherwise.

Let $1_{\sigma}(\alpha)$ be the infimum of lengths of closed curves representing $\alpha$. Obviously

$$
1_{\sigma}(\alpha)<c\|\alpha\|,
$$

where $c$ is a constant depending only on $\sigma$ and on the choice of the $\omega_{i}$. If $\alpha \neq 0$, the infimum is always realized by a closed geodesic.

For a geodesic $g^{\prime}$ which realizes the minimum of length in a homology class $\alpha$, one has

$$
\|\alpha\|<c_{1} \text { length } g^{\prime},
$$

where $c_{1}$ as before depends only on $\sigma$ and on the choice of the $\omega_{i}$.

Let us return to our initial almost uniformly distributed closed geodesic $g$. Let us denote $[g] \in H_{1}(M, Z)$ the homology class of $g$. If by chance $[g]=\beta$, the proof is finished. Otherwise, let $\alpha=\beta-[g]$ and let us construct a shortest closed geodesic $g^{\prime}$ representing $\alpha$. By the construction of $g$ it passes within $2 \delta$ of $g^{\prime}$, and by the shadowing lemma (see e.g. $[7, \S 2]$ ) one can construct a closed geodesic $g^{\prime \prime}$ which follows $g$ very closely, then follows $g^{\prime}$ very closely, and whose length is very close to the sum of the lengths of $g$ and $g^{\prime}$. The precise meaning of the words 'closely' and 'close' in the previous sentence is determined by $\delta$. Obviously

$$
\left[g^{\prime \prime}\right]=[g]+\left[g^{\prime}\right]=[g]+\beta-[g]=\beta \text {. }
$$


On the other hand, by (8),

$$
\text { length } g^{\prime}<c(\|g\|+\|\beta\|),
$$

and by our choice of $g$ it follows from (9) that $\left\|g^{\prime}\right\|<\varepsilon_{1}$ length $g$. Since $\beta$ is fixed and $g$ can be chosen arbitrarily long, we can also assume that $\|\beta\|<\varepsilon_{1}$ length $g$. Thus

$$
\text { length } g^{\prime}<2 c \varepsilon_{1} \text { length } g \text {. }
$$

Now, choosing $\varepsilon_{1}$ and $\delta$ sufficiently small from the beginning, one can guarantee that the averages of the functions $f_{1}, \ldots, f_{m}$ over $g^{\prime \prime}$ are arbitrarily close to those for $g$ and consequently are within $\varepsilon$ of the integrals

$$
\int_{S^{\sigma} M} f_{i} d \lambda_{\sigma}, \quad i=1, \ldots, m .
$$

\section{Geodesic and harmonic measures at infinity}

Let $\tilde{M}$ be the universal cover of the surface $M$. Any Riemannian metric $\sigma$ on $M$ has a natural lift to $\tilde{M}$ which we will denote by $\tilde{\sigma}$. If $\sigma$ is a metric of negative curvature, then the classes of $\tilde{\sigma}$-geodesics on $\tilde{M}$, asymptotic to each other in the positive direction, determine the circle at infinity which we will denote by $M(\infty)$. Since every two $\tilde{\sigma}$-geodesics which do not converge to each other exponentially in the positive direction diverge exponentially, the elements of the circle at infinity can also be described as classes of $\tilde{\sigma}$-geodesics which stay a bounded distance apart in the positive direction. This description shows that the circle at infinity for $\tilde{M}$ is defined canonically for all metrics of negative curvature on $M$. For, let $\sigma, \sigma^{\prime}$ be two such metrics. For any $\tilde{\sigma}$-geodesic $\gamma$ there exists exactly one $\tilde{\sigma}$-geodesic $\gamma^{\prime}$ which stays a bounded distance away from $\gamma$. Thus the points of the circles at infinity defined by $\sigma$ and $\sigma^{\prime}$, which are determined by geodesics asymptotic to $\gamma$ and $\gamma^{\prime}$ correspondingly, are naturally identified. The space $N=\tilde{M} \cup M(\infty)$ is provided with a natural topology which makes it homeomorphic to a closed disc.

The fundamental group $\Pi=\pi_{1}(M)$ acts on $\tilde{M}$ and preserves the lift of any Riemannian metric from $M$. This action is extended to an action by homeomorphisms of $N$.

The correspondence between $\tilde{\sigma}$ - and $\tilde{\sigma}^{\prime}$-geodesics described above, which associate $\gamma$ and $\gamma^{\prime}$, is obviously $\Pi$-equivariant and thus can be projected to $M$. Furthermore, one can extend it to a $\Pi$-equivariant map $G$ between the unit tangent bundles to $\tilde{M}$ for $\tilde{\sigma}$ and $\tilde{\sigma}^{\prime}$ which agrees with it (see e.g. [4]). The map $G$ may be made smooth along $\sigma$-geodesics, but it is not canonically defined and in general it does not preserve the time parameter for the geodesic flow, i.e. the length parameter along geodesics. In order for that to be possible, the metrics $\sigma$ and $\sigma^{\prime}$ have to have the same length spectrum. Theorem A (see $\S 2$ ) shows that it is also sufficient. The projection of the map $G$ to the unit tangent bundle of $M$ defines a continuous isotopic to identity orbit equivalence $g$ between the geodesic flows $\phi^{\sigma}$ and $\phi^{\sigma^{\prime}}$. Such an orbit equivalence is uniquely defined up to a variable shift along the orbits of $\phi^{\sigma}$. In particular, if $g$ is smooth along the orbits of that flow, then for every $\phi^{\sigma}$-invariant measure $\mu$ the class of the measure $g_{*} \mu$ is independent of a particular choice of $g$. Since $\psi_{t}=g^{-1} \phi_{t}^{\sigma} g$ is a flow which differs from $\phi_{t}^{\sigma^{\prime}}$ by a positive velocity 
change, the measure $g_{*} \mu$ is equivalent to a $\phi^{\sigma^{\prime}}$-invariant measure. In particular, since Liouville measure $\lambda_{\sigma}$ is ergodic with respect to the flow $\phi^{\sigma}$, the measure $g_{*} \lambda_{\sigma}$ is equivalent to an ergodic invariant measure for $\phi^{\sigma^{\prime}}$. Hence it is either equivalent to $\lambda_{\sigma}$ or is singular with respect to it. In [9] the following result is proved.

THEOREM B. Let $\sigma, \sigma^{\prime}$ be two metrics of negative curvature on a compact surface $M$. If there exists a continuous absolutely continuous isotopic to identity orbit equivalence between the geodesic flows $\phi^{\sigma}$ and $\phi^{\sigma^{\prime}}$, then the metrics $\sigma$ and $\sigma^{\prime}$ have the same geodesic length spectrum.

For each point $p \in \tilde{M}$ and each $\xi \in M(\infty)$ there exists exactly one semi-infinite $\sigma$-geodesic which starts at $p$ and is asymptotic to $\xi$. Let $v_{p}(\xi) \in T_{p}(\tilde{M})$ be the unit tangent vector at $p$ determining the geodesic and, conversely, for $v \in T_{p}(\tilde{M})$ let $\xi_{p}(v) \in M(\infty)$ be the equivlaence class of the geodesic beginning at $v$.

For any two points $p, q \in \tilde{M}$ the map

$$
\Phi_{p, q}=v_{p} \circ \xi_{p}: S_{p}^{\sigma} \tilde{M} \rightarrow S_{q}^{\sigma} \tilde{M}
$$

is a $C^{1}$ (but in general not $C^{2}$ ) diffeomorphism [5]. Thus a canonical $C^{1}$ structure is defined on $M(\infty)$ as the image of the standard $C^{1}$ structure on $S_{p}^{\sigma} \tilde{M}$ under the map $\xi_{p}$ for any $p \in \tilde{M}$. In particular, the $\xi_{p^{*}} \theta$, the images of Lebesgue measure $\theta$ on $S_{p}^{\sigma} \tilde{M}$, determine a class of equivalent measures at the circle at infinity which we will call the geodesic measure class at infinity.

Another way to produce a canonical measure class at infinity is to consider the Brownian motoion on $\tilde{M}$ starting at a point $p$. Almost every path of that motion converges to a point on $M(\infty)$. Thus for a Borel set $B \subset M(\infty)$ we can define the probability that the Brownian path beginning at $p$ converges to a point $\xi \in B$. This determines a measure $\eta_{p}$ on $M(\infty)$. For different initial points $p, q \in \tilde{M}$ the measures $\eta_{p}$ and $\eta_{q}$ are equivalent. These measures are called harmonic measures and their equivalence class the harmonic measure class at infinity.

THEOREM 4. If $\sigma$ is a metric of non-constant negative curvature on $M$, then geodesic and harmonic measure classes at infinity are mutually singular.

Proof. The first important observation is that since a Laplacian in dimension two is conformally invariant, the Brownian motions for conformally equivalent metrics have the same orbits and differ only by a time change. Hence harmonic measures for conformally equivalent metrics are the same. For a metric of constant negative curvature, harmonic and geodesic measure classes coincide. This becomes obvious if one considers $\tilde{M}$ as the standard Poincaré disc and takes the origin as the point $p$. Both the geodesic and the harmonic measures are rotationally invariant and therefore coincide with Lebesgue measure on the unit circle.

Proposition. Let $\sigma, \sigma^{\prime}$ be two metrics of negative curvature on $M$. Then either there exists a continuous absolutely continuous isotopic to identity orbit equivalence betwen the geodesic flows $\phi^{\sigma}$ and $\phi^{\sigma^{\prime}}$ or the geodesic measure classes for $\sigma$ and $\sigma^{\prime}$ are mutually singular. 
This is an almost immediate corollary of the fact that the correspondence between two transversals along the leaves of the weak-stable foliation is $C^{1}$ and hence is absolutely continuous.

Let us outline the original proof of theorem 4 which the author found in 1984. As before let $\sigma_{0}=\rho \sigma$ be the metric of constant negative curvature with $V_{\sigma_{0}}=V_{\sigma}$. Since the harmonic measure class of $\sigma$ coincides with the geodesic measure class for $\sigma_{0}$, then, unless the geodesic and harmonic measure classes of $\sigma$ are mutually singular, one deduces from the proposition, theorem $B$ and theorem $A$ that the geodesic flows $\phi^{\sigma}$ and $\phi^{\sigma_{0}}$ are conjugate via a diffeomorphism $g$. This obviously implies that $h_{\sigma}=h_{\sigma_{0}}$. Since the image $g_{*} \lambda_{\sigma}$ is an absolutely continuous $\phi^{\sigma_{0} \text {-invariant }}$ measure, it must coincide with $\lambda_{\sigma_{0}}$ so that $h_{0}^{\lambda}=h_{\sigma_{0}}^{\lambda}$. Thus we have $h_{\sigma}^{\lambda}=h_{\sigma_{0}}^{\lambda}=h_{\sigma_{0}}=h_{\sigma}$ and by theorem $1 \rho_{\sigma}=1$, i.e. $\rho \equiv 1$ and $\sigma=\sigma_{0}$.

Now we will present a self-contained proof of theorem 4 which does not depend on theorems A and B and follows very closely the arguments from the proof of theorem 1 presented in $\S 1$.

Proof. Let $g$ be an orbit equivalence between the geodesic flows $\phi^{\sigma}$ and $\phi^{\sigma_{0}}$ isotopic to identity. We assume that the map $g$ is smooth along the orbits of $\phi^{\sigma}$. As we already mentioned, the flow $\psi_{t}=g^{-1} \phi_{i}^{\sigma} g$ differs from $\phi^{\sigma_{0}}$ by a positive velocity change, i.e. if $\eta=d \phi_{t}^{\sigma_{0}} /\left.d t\right|_{t=0}$ is the vector field generating $\phi^{\sigma_{0}}$, then the flow $\psi_{t}$ is generated by the vector field $a \eta$, where $a$ is a positive scalar function. We still use the proposition in order to deduce that either the geodesic and harmonic measure classes for $\sigma$ are mutually singular or the orbit equivalence $g$ is absolutely continuous. In the latter case the measure $g_{*} \lambda_{\sigma}$ is absolutely continuous and is invariant with respect to the flow $\psi_{t}$. But the measure $a^{-1} \lambda_{\sigma_{0}}$ is also absolutely continuous $\psi_{t^{-}}$ invariant and ergodic, so that the two measures must be proportional. Now let us fix a large positive number $T$ and, as in the proof of theorem 1 , consider the set $S_{T}$ of all closed geodesics for $\sigma_{0}$ of length $\leq T$ which are almost uniformly distributed with respect to the measure $\lambda_{\sigma_{0}}$. As we have shown, those geodesics constitute the majority among all closed geodesics of length $\leq T$, so we can assume that card $S_{T}>$ $\frac{1}{2} P_{\sigma_{0}}(T)$.

Every geodesic $\gamma \in S_{T}$ after a reparametrization becomes a closed orbit $\gamma^{\prime}$ of the flow $\psi_{t}$, which is almost uniformly distributed with respect to Liouville measure $\lambda_{\sigma}$. Obviously, in making the meaning of 'almost uniformly distributed' precise, one has to use different constants for different cases.

On the other hand, since the map $g$ is isotopic to identity, the $\sigma$-geodesic $g^{-1} \gamma^{\prime}$ belongs to the same free homotopy class as $\gamma$. Thus, assuming that the average of the function $\rho^{-1 / 2}$ over all $\gamma \in S_{T}$ is close to $\rho_{\sigma}$, we deduce as in the proof of theorem 1 that the $\sigma$-length of $g^{-1} \gamma^{\prime}$ is less than $\left(\rho_{\sigma}+\varepsilon\right)$ times the $\sigma_{0}$-length of $\gamma$, where $\varepsilon$ is an arbitrarily small number fixed in advance.

Let $\mu_{T}$ be the average of uniform $\delta$-measures on all geodesics $g^{-1} \gamma^{\prime}$ for $\gamma \in S_{T}$. Since every such geodesic is almost uniformly distributed with respect to $\lambda_{\sigma}$, as $T \rightarrow \infty$ we obtain $\mu_{T} \rightarrow \lambda_{\sigma}$. Arguing as in (5), we obtain

$$
h_{\sigma}^{\lambda} \geq \varlimsup_{T \rightarrow \infty} \frac{\log \operatorname{card} S_{T}}{\left(\rho_{\sigma}+\varepsilon\right) T} \geq \lim _{T \rightarrow \infty} \frac{\log \frac{1}{2} P_{\sigma_{0}}(T)}{\left(\rho_{\sigma}+\varepsilon\right) T}=\left(\rho_{\sigma}+\varepsilon\right)^{-1} h_{\sigma_{0}} .
$$


Since $\varepsilon$ can be chosen arbitrarily small, by theorem 1 this is impossible unless $\rho_{\sigma}=1$, i.e. $\rho \equiv 1$ so that $\sigma=\sigma_{0}$.

Remark. Besides the two proofs of theorem 4 presented here, there are two more known proofs. One of them was found by Ledrappier [11] and is based on some entropy-like considerations for the Brownian motion and on the conformal equivalence. The other $([5]$, corollary 10.5$)$ is a corollary of the rigidity of the Godbillon-Vey class for weak-stable foliations for surfaces of constant negative curvature.

\section{Estimate of Cheeger isoperimetric constant}

As before let $\tilde{M}$ be the universal cover of $M$ and $\tilde{\sigma}$ be the natural lift of the metric $\sigma$ to $\tilde{M}$. The asymptotic Cheeger isoperimetric constant $C_{\sigma}$ is defined as the lower limit of the ratios of the length of a rectifiable closed Jordan curve on $\tilde{M}$ to the area bounded by the curve as the area goes to infinity. The usual definition of the isoperimetric constant does not include the assumption that the area goes to infinity. We include it to avoid a situation of a surface with a large relatively flat piece and many handles with large curvature.

If one fixes the length of the curve and considers the infimum with that restriction, it is easy to deduce from the fact that the metric $\tilde{\sigma}$ is a lift of a metric on a compact surface that the infimum is actually achieved. Then the following rather standard variational argument shows that any extremal curve has constant geodesic curvature and the value of that curvature is equal to the ratio in question.

Let $\Gamma$ be a closed curve on $\tilde{M}$ of length $l$ bounding a domain of area $V$ and provided with the length parameter $s$. Let $N(s)$ be the unit vector of exterior normal to $\Gamma$ and $H(s)=h(s) N(s)$ be the vector of geodesic curvature, so that $h(s)=|H(s)|$ is the geodesic curvature of $\Gamma$ at the point $s$. Furthermore, let $E$ be a vector field on $\Gamma$ which determines a smooth variation of $\Gamma$, i.e. a smooth one-parameter family of curves $\Gamma_{t}$ of length $l_{t}$, bounding the area $V_{t}$. Then an explicit calculation shows that

$$
\begin{aligned}
\frac{d}{d t}\left(\frac{l_{t}}{V_{t}}\right)_{t=0} & =\frac{1}{V} \int_{\Gamma}\langle H(s), E(s)\rangle d s-\frac{l}{V^{2}} \int_{\Gamma}\langle N(s), E(s)\rangle d s \\
& =\frac{1}{V} \int_{\Gamma}\left\langle H(s)-\frac{l}{V} N(s), E(s)\right\rangle d s .
\end{aligned}
$$

If $\Gamma$ is an extremal curve of length $l$, then the integral at the right-hand part of (10) is equal to zero for every smooth vector field $E$, so that $h(s)=l / V$. Thus $C_{\sigma}$ can be calculated by considering only smooth closed curves of constant geodesic curvature. In particular, this observation allows us to show that for a metric of constant negative curvature $-K^{2}$

$$
C_{\sigma}=K=\left(-2 \pi E / V_{\sigma}\right)^{1 / 2}=h_{\sigma}=h_{\sigma}^{\lambda}
$$

THEOREM 5. For an arbitrary metric $\sigma$ on a compact surface

$$
C_{\sigma} \leq \rho_{\sigma}\left(-2 \pi E / V_{\sigma}\right)^{1 / 2}
$$

Proof. As before let $\sigma_{0}=\rho \sigma$ be the metric of constant negative curvature such that $V_{\sigma_{0}}=V_{\sigma}$. Lift $\sigma$ and $\sigma_{0}$ to the metrics $\tilde{\sigma}$ and $\tilde{\sigma}_{0}$ on $\tilde{M}$. Take a large circle $C$ on $\tilde{M}$ 
with respect to the metric $\tilde{\sigma}_{0}$ as a test curve. The ratio of the $\tilde{\sigma}_{0}$-length of $C$ to the $\tilde{\sigma}_{0}$-area of its interior is close to $\left(-2 \pi E / V_{\sigma}\right)^{1 / 2}$.

Let us see what happens to the length and the area when we pass from $\tilde{\sigma}_{0}$ to $\tilde{\sigma}$. As before, the $\tilde{\sigma}$-length of $C$ is equal to its $\tilde{\sigma}_{0}$-length times the average of the function $\rho^{-1 / 2}$ over $C$ taken with respect to the $\tilde{\sigma}_{0}$-length parameter. The last quantity is close to $\int_{M} \rho^{-1 / 2} d \mu_{0}$ which, as we already mentioned, is equal to $\rho_{\sigma}$.

The reason for that is that the projection of a circle to $M$ converges to a horocycle when the radius goes to infinity, and the projection of the normalized arc length measure to $S^{\sigma} M$ weakly converges to an invariant measure for the horocycle flow. But the only invariant measure for that flow is Liouville measure.

In order to understand how the area changes, let us introduce the following coordinates inside the disc $D$ bounded by the circle $C$. Let $r$ be the $\sigma_{0}$-arc length parameter along radial geodesics, and $s$ be the $\sigma_{0}$-arc length parameter along the concentric circles. Let $l(r)$ be the $\sigma_{0}$-length of the circle of radius $r$. Since the area element in $(r, s)$ coordinates is simply $d s d r$, the $\sigma_{0}$-area of the disc $D$ of radius $R$ is equal to

$$
A_{0}=\int_{0}^{R} \int_{0}^{l(r)} d s d r=\int_{0}^{R} l(r) d r
$$

and the $\sigma$-area is equal to

$$
A=\int_{0}^{R} \int_{0}^{I(r)} \tilde{\rho}^{-1}(s, r) d s d r
$$

where $\tilde{\rho}$ is the lift of $\rho$ to $\tilde{M}$.

Since the length $l(r)$ grows exponentially with $r$, the dominant contribution to both expressions (11) and (12) comes from the integration between $\dot{R} / 2$ and $R$. But for a large $R$ and for every $r: R / 2 \leq r \leq R$ the projection of any $\tilde{\sigma}_{0}$-circle of radius $r$ is already almost uniformly distributed with respect to $\lambda_{\sigma_{0}}$ (see above). Thus for $R / 2 \leq r \leq R$

$$
\left|\int_{0}^{l(r)} \tilde{\rho}^{-1}(s, r) d s-l(r) \int_{M} \rho^{-1} d \mu_{\sigma_{0}}\right|
$$

is small but

$$
\int \rho^{-1} d \mu_{\sigma_{0}}=\frac{V_{\sigma}}{V_{\sigma_{0}}}=1,
$$

so that the ratio $A / A_{0}$ is close to one.

Thus the ratio of the $\sigma$-length of $C$ and the $\sigma$-area of $D$ is close to $\rho_{\sigma}\left(-2 \pi E / V_{\sigma}\right)^{1 / 2}$ and the Cheeger constant, being the lower limit of all such ratios, should be less than or equal to that quantity.

\section{Comparison of various measures of deviation from constant negative curvature}

Several important numerical invariants of a Riemannian metric $\sigma$ can be interpreted as measuring the deviation of $\sigma$ from metrics of constant negative curvature. These characteristics come from three different sources: geometry, complex analysis and dynamics. It is natural to consider normalized quantities which do not change when 
a metric is multiplied by a scalar constant. We will mention five such characteristics. Some of them make sense only for metrics of negative curvature, others for arbitrary metrics.

(1) The most straightforward geometric characteristic for a metric of non-positive curvature with the curvature function $-K^{2}(x), x \in M$, is the normalized average of the square root of the absolute value of the curvature

$$
k_{\sigma}^{N} \stackrel{\text { def }}{=} \frac{\int_{M} K(x) d \mu_{\sigma}(x)}{\left(\int_{M} K^{2}(x) d \mu_{\sigma}(x)\right)^{1 / 2}}=\frac{\int_{M} K(x) d \mu_{\sigma}(x)}{\left(-2 \pi E / V_{\sigma}\right)^{1 / 2}}
$$

(2) Another geometric characteristic is the normalized Cheeger constant

$$
C_{\sigma}^{N} \stackrel{\text { def }}{=} \frac{C_{\sigma}}{\left(-2 \pi E / V_{\sigma}\right)^{1 / 2}}
$$

Unlike the previous one, it makes sense for an arbitrary metric.

(3) The natural measure of deviation associated with conformal structure is the conformal coefficient $\rho_{\sigma}$, which is also defined for an arbitrary metric.

(4) The normalized topology entropy

$$
h_{\sigma}^{N} \stackrel{\text { def }}{=} \frac{h_{\sigma}}{h_{\sigma_{0}}}=\frac{h_{\sigma}}{\left(-2 \pi E / V_{\sigma}\right)^{1 / 2}}
$$

is a natural dynamical characteristic defined for an arbitrary metric.

(5) For metrics without focal points one can also consider the normalized Liouville entropy

$$
h_{\sigma}^{\lambda, N} \stackrel{\text { def }}{=} \frac{h_{\sigma}^{\lambda}}{h_{\sigma_{0}}^{\lambda}}=\frac{h_{\sigma}^{\lambda}}{\left(-2 \pi E / V_{\sigma}\right)^{1 / 2}} .
$$

If $\sigma$ is a metric of constant negative curvature, all five quantities are equal to one. For all other metrics the following inequalities hold:

$$
\begin{gathered}
k_{\sigma}^{N}<h_{\sigma}^{\lambda, N}<\rho_{\sigma}<1<h_{\sigma}^{N} \\
\bigvee \\
C_{\sigma}^{N}
\end{gathered}
$$

Each inequality holds whenever both quantities are defined.

All these inequalities except for the one on the far left have been discussed in the present paper. The remaining one is due to Manning [12] and Sarnak [14] and is obtained by studying the Riccati equation and using the Pesin entropy formula.

The only two remaining open questions are the relationships between the $C_{\sigma}^{N}$ on one side and the $k_{\sigma}^{N}$ and $h_{\sigma}^{\lambda, N}$ on the other. Positive solution of the following conjecture would transform (13) into a linearly ordered chain of inequalities.

Conjecture (M. Anderson). For any metric of negative curvature on a compact manifold

$$
h_{\sigma}^{\lambda} \leq C_{\sigma}
$$


In a somewhat different direction, let us mention that theorem 4 can be sharpened in the following way. Let $H_{\sigma}$ be the Hausdorff dimension of harmonic measures with respect to the $C^{1}$-structure defined by the maps $\xi_{p}$. Then for every metric $\sigma$ of non-constant negative curvature $H_{\sigma}<1$. Thus the number $H_{\sigma}$ measures how singular the harmonic measures are with respect to the geodesic ones. Relations between $H_{\sigma}$ and the five quantities mentioned above are not clear at this point.

Let us conclude this discussion with a remark on two inequalities on the left of (13). The conformal coefficient $\rho_{\sigma}$ is estimated from below through the area and the injectivity radius ([8], theorem 3.4$)$. Having this in mind, let us consider two examples:

(1) A metric of non-positive curvature with area one and injectivity radius bounded away from zero, which is flat or almost flat everywhere, except for a small neighbourhood of a finite set of points: for such a metric $k_{\sigma}^{N}$ is obviously very small. A study of the Riccati equation and the ergodicity of the geodesic flow shows that the metric entropy with respect to Liouville measure is also very small. Thus $k_{\sigma}^{N}<h_{\sigma}^{\lambda, N} \ll \rho_{\sigma}$ because $\rho_{\sigma}$ is bounded away from zero.

(2) All properties of the metric remain the same except for the distribution of the negative curvature. It is concentrated near a curve; e.g. we can consider two flat tori with a round hole of fixed size in each connected by a very narrow 'collar' of negative curvature. In this case $k_{\sigma}^{N}$ is still very small. However, $h_{\sigma}^{\lambda}$ is bounded away from zero so that we have $k_{\sigma}^{N} \ll h_{\sigma}^{\lambda, N}<\rho_{\sigma}$.

Acknowledgements. The first version of this paper was written during a visit to Instituto de Matemáticas, Universidad Nacional Autónoma de México, which was a part of the Semester in Dynamical Systems organized by Xavier Goméz-Mont and José A. Seade. In a way, the paper is a spin-off of the short course in Anosov systems, surfaces of negative curvature and characteristic classes of foliations given at UNAM in April 1986. I would like to thank the organizers of the Semester in Dynamical Systems for the invitation to visit UNAM and for their warm hospitality.

I am indebted to the referee who pointed out that without negative curvature or a similar assumption the geodesic length spectrum does not determine a metric even within a conformal class.

This work was partially supported by NSF Grant DMS 85-14630.

\section{REFERENCES}

[1] R. Bowen. Periodic orbits for hyperbolic flows. Amer. J. Math. 94 (1972), 1-30.

[2] K. Burns \& A. Katok in collaboration with W. Ballmann, M. Brin, P. Eberlein \& R. Osserman. Manifolds with non-positive curvature. Ergod. Th. \& Dynam. Sys. 5 (1985), 307-317.

[3] J. Cheeger. A lower bound for the smallest eigenvalue of the Laplacian. In Problems in Analysis, ed. R. C. Gunning. Princeton University Press, Princeton, NJ (1970), 195-199.

[4] M. Gromov. Three remarks on geodesic dynamics and fundamental group. Preprint.

[5] S. Hurder \& A. Katok. Differentiability, rigidity and Godbillon-Vey classes for Anosov flows. Preprint.

[6] A. Katok. Lyapunov exponents, entropy and periodic orbits for diffeomorphisms. Publ. Math. IHES 51 (1980), 137-173. 
[7] A. Katok. Dynamical systems with hyperbolic structure. AMS Trans. 116 (1981), 43-95.

[8] A. Katok. Entropy and closed geodesics. Ergod. Th. \& Dynam. Sys. 2 (1982), 339-367.

[9] A. Katok. Geodesic length spectrum and measures at infinity for metrics of negative curvature. To appear.

[10] W. Klingenberg, D. Gromoll \& W. Meyer. Riemannische Geometrie in grossen. Springer-Verlag, Berlin (1968).

[11] F. Ledrappier. Propriété de Poisson et courbure négative. Preprint.

[12] A. Manning. Curvature bounds for the entropy of the geodesic flow on a surface. J. Lond. Math. Soc. 24 (1981), 351-357.

[13] G. A. Margulis. One some applications of ergodic theory to the study of manifolds of negative curvature. Funct. Anal. Appl. 3 (1969), 69-90.

[14] P. Sarnak. Entropy estimates for geodesic flows. Ergod. Th. \& Dynam. Sys. 2 (1982), 513-524.

[15] M. Schiffer \& D. C. Spencer. Functionals on Finite Riemannian Surfaces. Princeton University Press, Princeton, NJ (1954). 